\title{
Erratum to: Hodgkin's lymphoma associated with paraneoplastic cerebellar degeneration in children: a case report and review of the literature
}

\author{
Serdal Gungor ${ }^{1} \cdot$ Betul Kilic $^{1} \cdot$ Mujgan Arslan $^{1} \cdot$ Unsal Ozgen $^{2} \cdot$ Josep Dalmau $^{3}$
}

Published online: 27 April 2017

(C) Springer-Verlag Berlin Heidelberg 2017

\section{Erratum to: Childs Nerv Syst}

DOI 10.1007/s00381-016-3284-y

On author's request, the name of Prof. Josep Dalmau has been removed from the author list. Despite Prof. Dalmau's support to the diagnosis part of the study, the co-authors acknowledge that he was unaware of the article publication and he did not approve the inclusion of his name in the authors' list.

Acknowledgements We are grateful to Prof. Dr. Josep Dalmau for his great contribution in the diagnosing part of this study.

The online version of the original article can be found at http://dx.doi. org/10.1007/s00381-016-3284-y

Betul Kilic

betulklc82@gmail.com

1 Faculty of Medicine, Department of Pediatric Neurology, Inonu University, Malatya, Turkey

2 Faculty of Medicine, Department of Pediatric Hematology, Inonu University, Malatya, Turkey

3 University of Barcelona, Catalan Institution of Research and Advanced Studies (ICREA) and Biomedical Research Institute August Pi i Sunyer (IDIBAPS), Hospital Clinic, Barcelona, Spain 